\title{
Aplicación del proceso de atención de enfermería basado en el modelo teórico de Virginia Henderson, en el centro gereatrico “Amawta Wasi Samay" Guaranda-Bolívar.
}

Application of the process of nursing attention based on the theoretical model of Virginia Henderson for elders from the center home "Amawta Wasi Samay", Guaranda-Bolivar.

Lourdes Maribel Cabascango Allauca. ${ }^{1}$, Ana Gabriela Hinojosa Chariguamán. ${ }^{2}$, Lourdes Morayma Remache Agualongo. ${ }^{3}$ \& María Humbelina Olalla García. ${ }^{4}$

\begin{abstract}
.
DOI: https://doi.org/10.33262/cienciadigital.v3i1.250

Nursing is considered an integrating discipline of support to the health area, with a great social impact that constantly expands its functions to meet the needs and improve patient care. The present investigation aims to: Apply the Process of Nursing Care based on the theoretical model of Virginia Henderson, in the elderly of the home center "Amawta Wasi Samay". Method: It is a descriptive, prospective, documentary, field and cross-sectional study, the collection of the information was achieved through a diagnostic survey to the nursing staff identifying the knowledge and application of the PAE / Virginia Henderson model; In addition to 2 observation cards one to characterize the current profile of the older adult and another to determine what nursing interventions are performed in the home center, in addition to a survey to assess the perception of the elderly in relation to the care received. Results: $48 \%$

\footnotetext{
${ }^{1}$ Universidad Estatal de Bolívar, Facultad Ciencias de la Salud y del Ser Humano, Carrera de Enfermería, Bolívar, Ecuador, maribelcabascango1995@hotmail.com

${ }^{2}$ Universidad Estatal de Bolívar, Facultad Ciencias de la Salud y del Ser Humano, Carrera de Enfermería, Bolívar, Ecuador, gabriela.hnj@gmail.com

${ }^{3}$ Universidad Estatal de Bolívar, Facultad Ciencias de la Salud y del Ser Humano, Carrera de Enfermería, Bolívar, Ecuador, morayma_remache@ @otmail.com

${ }^{4}$ Universidad Estatal de Bolívar, Facultad Ciencias de la Salud y del Ser Humano, Carrera de Enfermería, Bolívar, Ecuador, mia_og2007@yahoo.es
} 
of the nursing staff know the PAE, but do not perform it in their daily work, $60 \%$ do not identify the Henderson model. $42 \%$ of nursing staff focus their care on the satisfaction of the biological component. $60 \%$ of older adults report that they feel satisfied with nursing interventions. Conclusions: The PAE and the assessment and care guide based on the Virginia Henderson Model were elaborated and applied, serving as a support tool when evaluating and providing care to the elderly.

Keywords: Nursing Care Process, Virginia Henderson, Interventions, Elderly Care.

\section{Resumen.}

La enfermería es considerada como una disciplina integradora de apoyo al área de la salud, de gran impacto social que constantemente amplía sus funciones para satisfacer las necesidades y mejorar el cuidado del paciente. La presente investigación tiene como objetivo: Aplicar el Proceso de Atención de Enfermería basado en el modelo teórico de Virginia Henderson, en los adultos mayores del centro hogar "Amawta Wasi Samay”. Método: Es un estudio descriptivo, prospectivo, documental, de campo y de corte transversal, la recolección de la información se logró a través de una encuesta diagnóstica al personal de enfermería identificando el conocimiento y la aplicación del PAE/modelo de Virginia Henderson; además de 2 fichas de observación una para caracterizar el perfil actual del adulto mayor y otra para determinar que intervenciones de enfermería se realizan en el centro hogar, además de una encuesta para evaluar la percepción de los adultos mayores en relación a los cuidados recibidos. Resultados: El 48\% del personal de enfermería conoce el PAE, pero no lo realizan en su trabajo diario, el $60 \%$ no identifica el modelo de Henderson. El $42 \%$ de personal de enfermería enfocan su cuidado a la satisfacción del componente biológico. El 60\% de adultos mayores refieren que se sienten satisfechos con las intervenciones de enfermería. Conclusiones: Se elaboró y aplico el PAE y la guía de valoración y cuidado basado en el Modelo de Virginia Henderson, sirviendo como herramienta de apoyo a la hora de valorar y brindar cuidados al adulto mayor.

Palabras claves: Proceso de Atención de Enfermería, Virginia Henderson, Intervenciones, Cuidados del Adulto Mayor.

\section{Introducción.}

El proceso de atención de enfermería y la valoración geriátrica aplicado a través de modelo teórico de Virginia Henderson es una herramienta esencial que permite detectar y cuantificar los problemas de salud que afectan a este grupo vulnerable, en consecuencia, los cuidados van encaminados a ese mantenimiento de la vida, a través de la promoción de la higiene, la adecuada alimentación, el vestido y en general todas las medidas que hacen la vida más agradable. El cuidado va dirigido a mantener la salud del adulto mayor en todas sus 
dimensiones: física, mental y social. La enfermera evalúa las necesidades de ayuda de la persona teniendo en cuenta sus percepciones y complejidad.

La presente investigación aborda dos variables muy bien definidas, la primera aborda el proceso de atención de enfermería basado en el Modelo Teórico de Virginia Henderson como una metodología científica, sistemática y coordinada que los profesionales de enfermería deben aplicar, mientras que la segunda se enfoca en el cuidado del adulto mayor. El envejecimiento es un proceso que contempla una de las etapas de ciclo de vida del ser humano y con ello ocurre varias modificaciones fisiológicas, psicológicas y psicosociales. Un adulto mayor se considera a partir de los 65 años o más, siendo uno de los grupos más vulnerables de nuestra sociedad, por lo que requieren de apoyo total o parcial para satisfacer sus necesidades y disfrutar de lo que queda de vida con mayor tranquilidad. En este contexto, es donde el profesional de enfermería contribuirá al cuidado transdisciplinario. Virginia Henderson, enfermera que realizó grandes aportes a la enfermería como profesión, desarrollo su modelo basado en las 14 necesidades, donde el papel de la enfermera es la realización (suplencia o ayuda), que las personas no pueden realizar en un determinado momento de su ciclo vital. La aplicación de este modelo y el proceso de atención de enfermería, tiene una estrecha relación ya que mediante este proceso cíclico y sistemático se brindará una atención integral partiendo desde una valoración exhaustiva de problemas y formulación de diagnósticos de enfermería para posteriormente planificar, ejecutar y evaluar cuidados encaminadas a satisfacer las necesidades de los adultos mayores, fomentando su recuperación y nivel de independencia.

\section{Materiales y Métodos.}

Se trata de un estudio descriptivo correlacional, partiendo de un análisis donde se compara un método existente en un lugar determinado que permite detallar el Modelo Teórico de Virginia Henderson y el desarrollo del Proceso de Atención de Enfermería basado en la satisfacción de las 14 necesidades y el logro de la independencia del adulto mayor, es prospectivo por que se realizó con datos obtenidos a propósito de la investigación acerca de las intervenciones de enfermería en el centro hogar "Amawta Wasi Samay", el centro hogar alberga a 33 adultos mayores con diferentes problemas de salud, determinada con la aplicación de una ficha de observación donde se estableció el perfil actual de salud, por lo que se consideró la aplicación de una encuesta a 15 adultos mayores en pleno uso de sus facultades mentales, permitiendo evaluar la percepción de las intervenciones de enfermería brindadas, así como también una encuesta diagnóstica aplicada a 5 personas que forman parte del equipo de salud de enfermería para identificar conocimientos sobre el PAE y el modelo de Virginia Henderson más una ficha diagnóstica para determinar que intervenciones brindan a los adultos mayores. El resultado obtenido se presenta en gráficos estadísticos tipo pasteles y barras que facilito su interpretación y análisis. 


\section{Resultados.}

Gráfico 1. Resultados diagnósticos sobre la aplicación del Proceso de Atención de Enfermería y el conocimiento del modelo de Virginia Henderson al personal de enfermería del centro hogar “Amawta Wasi Samay”.

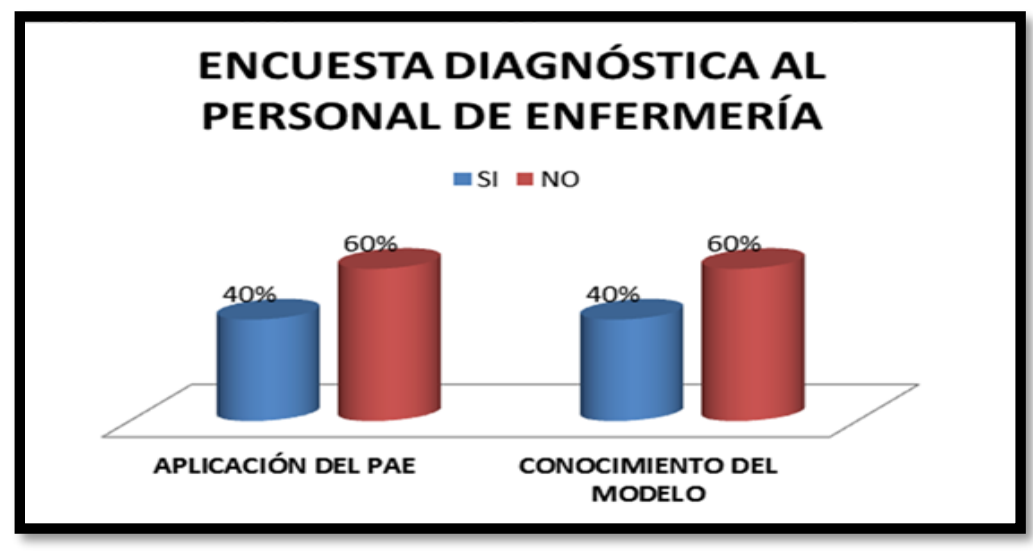

Elaborado por: Grupo de investigación

Los datos muestran que un $40 \%$ del personal de enfermería que labora en esta institución aplica el PAE, mientras que un $60 \%$ indica que no lo realiza, sin embargo, no existe evidencia documentada del porcentaje que refiere que si cumple; en relación al conocimiento del Modelo teórico de Virginia Henderson se obtuvo que el $60 \%$ no conoce y el $40 \%$ indica que, si lo conoce, sin embargo, no lo aplican en este grupo etareo.

Gráfico 2. Resultados diagnósticos sobre la aplicación del Proceso de Atención de Enfermería y el conocimiento del modelo de Virginia Henderson al personal de enfermería del centro hogar “Amawta Wasi Samay”.

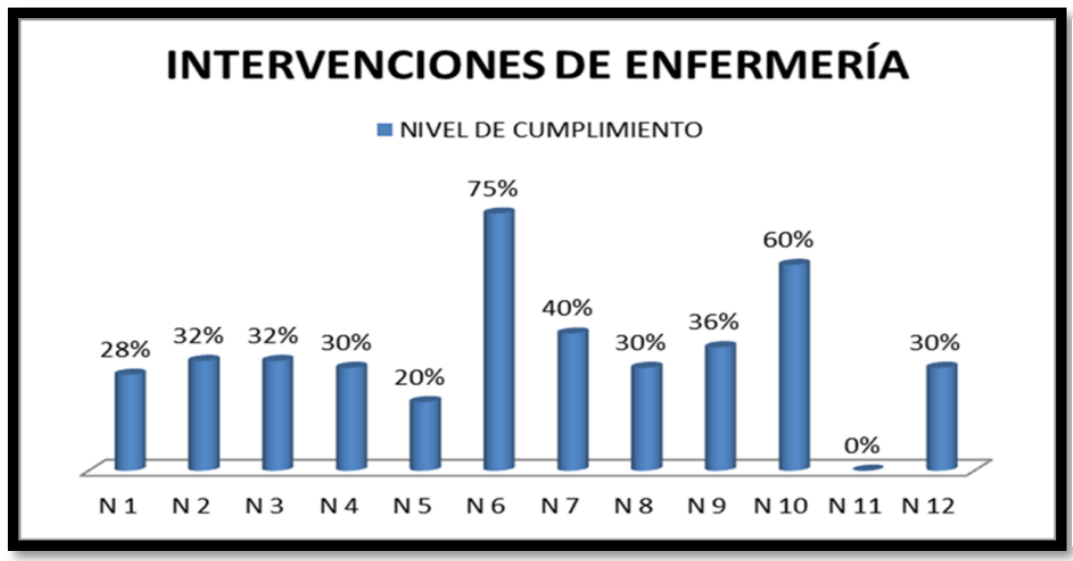

I. Respirar normalmente.

2. Comer y beber adecuadamente.

3. Eliminar por todas las vías corporales

4. Moverse y mantener posturas adecuadas.

5. Dormiry descansar.

6. Escoger la ropa adecuada, vestirse y desvestirse

7. Mantener la temperatura corporal dentro de los límites normales, adecuando la ropa y modificando el ambiente.

8. Mantener la higiene corporal y la integridad de la piel.

9. Evitar peligros ambientales y evitar lesionar a otras personas.

10. Comunicarse con los demás expresando emociones, necesidades, te-

mores u opiniones.

II. Vivir de acuerdo con los propios valores y creencias.

12. Ocuparse en algo de tal forma que su labor tenga un sentido de realización personal.

I3. Participar en actividades recreativas.

14. Aprender, descubrir o satisfacer la curiosidad que conduce a un desamo-

llo normal ya usar los recursos disponibles.

Elaborado por: Grupo de investigación 
Los porcentajes alcanzados en cuanto al cumplimiento de las 14 necesidades una vez aplicadas las intervenciones de enfermería basadas en el modelo teórico de Virginia Henderson generaron satisfacción y un mínimo de independencia en este grupo poblacional se obtuvo que las necesidades de mayor nivel de cumplimiento, siendo de las más destacadas las siguientes son: Necesidad 6: Escoger ropa adecuada, vestirse y desvestirse (Vestirse/desvestirse), con un $75 \%$

- Necesidad 10: Comunicarse con los demás expresando emociones, necesidades, temores u opciones. (Comunicación), con un $60 \%$

- Necesidad 7: Mantener la temperatura dentro de los límites normales adecuando la ropa y modificando el entorno. (Termorregulación), con un $40 \%$

- Mientras que la necesidad de menor satisfacción es Vivir de acuerdo con sus propios valores y creencias (Valores/creencias), con un nivel de cumplimiento de $0 \%$

Evidenciándose que el personal de enfermería solo cubre los requerimientos en relación al componente biológico, olvidándose del componente psicológico y espiritual declinando el factor biopsicosocial de todo individuo.

Tabla 1. Ficha de Observación/Valoración al Adulto Mayor, Centro Hogar “Amawta Wasi Samay

\begin{tabular}{|c|c|c|}
\hline \multicolumn{3}{|c|}{ FICHA DE OBSERVACIÓN AL ADULTO MAYOR } \\
\hline Capacidad funcional & Frecuencia & Porcentaje \\
\hline Dependiente & 22 & $67 \%$ \\
\hline Independiente & 11 & $33 \%$ \\
\hline Total & 33 & $100 \%$ \\
\hline Discapacidad & Frecuencia & Porcentaje \\
\hline Motriz & 6 & $18 \%$ \\
\hline Visual & 8 & $24 \%$ \\
\hline Auditiva & 3 & $9 \%$ \\
\hline Mental & 6 & $18 \%$ \\
\hline Ninguna & 10 & $31 \%$ \\
\hline Total & 33 & $100 \%$ \\
\hline
\end{tabular}

Elaborado por: Grupo de investigación

De la población Adulta mayor del centro hogar Amawta Wasi Samay, se observó que el 67\% son hombres y el $33 \%$ son mujeres, en lo que concierne a estado civil el $91 \%$ es soltero, seguido del $6 \%$ casado y el $3 \%$ viudo, mientras que en etnia se refiere el $67 \%$ son mestizos, el 33\% son indígenas, y afro ecuatorianos no se identificó por lo tanto no representa ningún porcentaje. 
En relación a discapacidad se observa que el $10 \%$ no presenta ningún tipo de discapacidad, $8 \%$ tienen una discapacidad visual, $6 \%$ motriz, $6 \%$ mental y un 3\% auditiva. En lo que a capacidad funcional se refiere el $67 \%$ es dependiente y el $33 \%$ es independiente, el mismo que se relaciona con el tipo de movilidad en el cual $88 \%$ es ambulatorio, el 9\% permanece en una silla y el $3 \%$ esta encamado, los mismos que para su movilización usan dispositivos como sillas de ruedas el $7 \%$, bastón el $2 \%$, andador el 3\%, muletas el $0 \%$ y el $64 \%$ no utilizan ningún dispositivo de apoyo.

Gráfico 3. Resultados de la percepción de los residentes sobre la atención obtenida por el personal de enfermería.



Elaborado por: Grupo de investigación

Para la obtención de estos resultados se escogió una muestra de 15 adultos mayores basándonos en la categorización del grupo de estudio obtenida a través de la ficha de observación encontraban pues esta población se encuentran en pleno uso de sus facultades cognitivas completas y podían formular su percepción sobre los cuidados obtenidos por el personal de enfermería.

Así de los 15 adultos mayores evaluados el $60 \%$ dice que siempre se siente satisfecho, mientras que el $40 \%$ indica que a veces. Podemos deducir que tienen una percepción aceptable a la atención brindada por el personal de enfermería, pues indicaron que son ellas las que se preocupan y están constantemente con ellos, mientras que sus familiares los abandonaron a su suerte y no les visitan. De allí se denota la importancia de esta noble profesión.

\section{Conclusiones}

- Con este trabajo investigativo se ha llegado a la conclusión que el personal de enfermería conoce, pero no aplica el Proceso de Atención de enfermería y que el cuidado brindado a los adultos mayores del Centro Hogar Amawta Wasi Samay es rutinario, empírico y sin sustento científico. 
- Según datos obtenidos de la ficha de observación aplicada al personal de enfermería se evidenció que no se brindan intervenciones de manera individualizada según los requerimientos del adulto mayor, factor que declina el enfoque biopsicosocial del individuo por la insatisfacción de las 14 necesidades básicas denotadas en el modelo de Virginia Henderson.

- Al ejecutar la guía de observación para caracterizar el perfil del adulto mayor, se pudo determinar que no existe un registro o reporte de enfermería evidente en las historias clínicas, de las intervenciones brindadas a los adultos mayores.

- En base a los resultados analizados de la encuesta diagnóstica, aplicada a los adultos mayores, se puede concluir que la mayoría tiene una percepción favorable sobre los cuidados de enfermería brindados en relación al componente biológico, mientras que en el componente social y psicológico determinan una percepción limitada debido a que no incentivan a la inclusión y realización de actividades recreativas que permitan disminuir sus tensiones, sin embargo describen que valoran el labor y trabajo de sus cuidadoras porque son ellas quienes velan por su bienestar.

\section{Referencias bibliográficas.}

Abizanda, P., \& Leocadio, R. (2015). Valoración Geriátrica. En Tratado de medicina geriátrica - (págs. 164-182). Barcelona, España : Elsevier.

Álvarez, M., Arkáute, I., Belaustegi, A., Chaparro, S., Erice, A., Pilar, M., . . . Santos, I. (Mayo de 2004). seeiuc.org. Obtenido de Sociedad Española de Enfermería Intensiva y Unidades Coronarias: http://www.seeiuc.com/profesio/criticos.pdf

Álvarez, P., \& Martínez, D. (2008). Valoración integral del adulto mayor. En Guias clínicas Geronto Geriatricas de atención primaria de salud para el adulto mayor (págs. 1983). Quito, Ecuador: normalización S.N.S.

Armijo, P. P. (12 de octubre de 2012). Aplicación de la teoría de Henderson y su aproximación al cuidado avanzado en enfermería en un servicio de pedatría. MEDWAVE. Obtenido de http://www.medwave.cl/link.cgi/Medwave/Revisiones/Analisis/5548

Artiga, B., Company, R., \& Sacristan, C. (29 de Noviembre de 2012). diposit.ub.edu. Obtenido de Dipósit Digital de la Universidad de Barcelona : http://www.diposit.ub.edu/dspace/vitectream/2445/34284/1/34/284.pdf

Barreto, J., \& Giron, J. (30 de Abril de 2014). La situación actual y expectativas de la población adulta mayor: un desafio para la salud. Recuperado el 3 de Junio de 2017, de Universidad Católica de Manizales-biblioteca: http://hdl.handle.net/10839/702 
Correa, E., Verde, E., \& Rivas, J. (2016). Descripción de la tipología de las 14 necesidades. En Valoración de enfermería basada en la filosofía de Virginia Henderson (págs. 25-45). México: Casa abierta al tiempo.

Fernández, M. (16 de Marzo de 2011). Unican Bases Históricas y Teóricas de la Enfermería. Recuperado el 9 de Junio de 2017, de Universidad de Cantabria : http://ocw.unican.es/ciencias-de-la-salud/bases-historicas-y-teoricas-de-laenfermeria/materiales-de-clase-1/Enfermeria-Tema11(II).pdf

Gallegos, Rocio. (08 de 04 de 2012). problemática social de los adultos mayores. Recuperado el 13 de 06 de 2017, de Grupoam: http://problematicaadultosmayores.blogspot.com/2012/04/definicion-de-adultomayor-rocio.html

García, M., \& Martínez, R. (2012). Procesos de envejecer. En Enfermería y envejecimiento (pág. 9). Barcelona , Eapaña: Elsevier.

Gispert, C. (2014). Modelo conceptual de Virginia Henderson. En C. Gispert, Manual de enfermería (pág. 856). México: Océano Centrúm.

Gonzalez, J., Estrada, I., Belaustegi, A., Chaparro, S., Criado, A., Pilar, M., . . Santos, I. (2004). Modelo conceptual de Virginia Henderson. En Guía de Práctica ClínicaCuidados críticos de Enfermería (págs. 23-31). Txagorritxu: Evagraf, S. Coop.

Ilustre Colegio Oficial de Enfermeria de Jaén. (2010). Modelo de cuidados de Virginia Henderson. En Proceso enfermero desde el modelo de cuidados de Virginia Henderson y los lenguajes NNN (Primera ed., págs. 18-33). Jaén, España: Sefarad.

Lozano Zetina Maria Guadalupe. (23 de marzo de 2015). redalyc.org Conceptualización del proceso envejecimiento. Obtenido de Red de Revistas Cientificas de America Latina y el Caribe, España y Portugal: http://www.redalpyc.org/pdf/112/11201903.pdf

Marrín, J. M. (28 de Noviembre de 2005). mpsp.webs.uvigo.es El envejecimiento. Obtenido de Área de Medicina Preventiva e Saúde Pública : http://mpsp.webs.uvigo.es/rev031/envejecimiento-03-1.pdf

Minchola, B. (2014). El envejecimiento y sus problemas . En Cuidados de Enfermeria en el adulto mayor (págs. 10-11).

Ministerio de Inclusion Economica y Social . (s.f.). inclusion.gob.ec DIreccion Poblacion Adulta Mayor. Obtenido de Ministerio de Inclusion Economica y social: http://www.inclusion.gob.ec/direccion-adulta-mayor 
Montesinos, G., Ortega, M., Leija, C., Quintero, M., Cruz, G., \& Suárez, M. (2011). Validación de un instrumento de valoración de enfermería cardiovascular con el enfoque de Virginia Henderson. Enfermería Cardiológica, XIX, 17-18.

Morfi, L. R. (2017). Atención del personal de enfermería en la gerontología comunitaria en cuba. Scielo-revista cubana de enfermería, 3-11.

Murriener, A., \& Raile, M. (2005). Conceptos principales y definiciones. En Modelos y teorias en enfermeria (págs. 101, 102). Madrid, España: ELSEVIER.

Organización Panamericana de la Salud. (1993). Cambios Fisicos y sus consecuencias en la Vejez. En E. Ansola (Ed.), Enfermeria Gerontológica (pág. 15 a la 22).

Pilar, M. G., \& Ballesteros, E. (2006). Cambios inherentes al proceso de envejecimiento. En Enfermería Geriátrica (Segunda ed., págs. 27-32). Barcelona, España: Elsevier.

Portillo Streempel, J., Espinosa Almendro, J., \& Muñoz Cobos , F. (2005). Clasificando a las personas mayores. Una visión dinámica . Sociedad Andaluza de Medicina Familiar y Comutario, 167, 168.

Potter, P., \& Griffin, A. (2007). Fundamentos de Enfermeria (5ta ed., Vol. 1). Madrid, España: Elsevier.

Povedano, M. (2012). Las necesidades básicas en el anciano. En Enfermería Geriátrica y Gerontológica (págs. 4-31).

Tabloski, P. (2010). Cambios Fisiologicos en el envejecimiento. En M. M. Romo (Ed.), Enfermeria Gerontológica (segunda ed., págs. 349, 449, 489, 490, 491, 539, 540, 579, 578, 669, 671, 709, 710, 739, 791). Madrid, España: Pearson.

Vera, D. M. (2017). Significado de la calidad de vidad del adulto mayor. Scielo, peru-anales de la Facultad de Medicina, 288, 289.

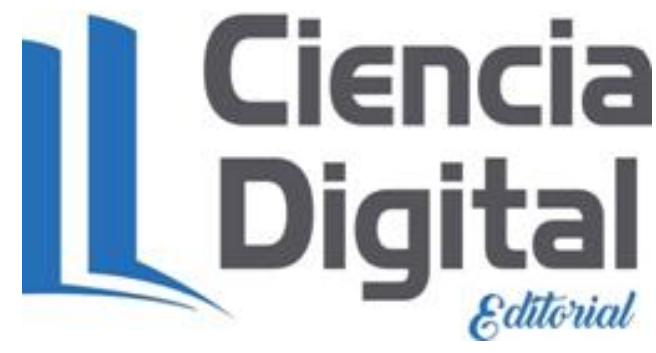




\section{Para citar el artículo indexado.}

Cabascango L., Hinojosa A., Remache L. \& Olalla M. (2019). Aplicación del proceso de atención de enfermería basado en el modelo teórico de Virginia Henderson, en el centro gereatrico "Amawta Wasi Samay" Guaranda-Bolívar. Revista electrónica Ciencia Digital 3(1), 54-63. Recuperado desde:

http://cienciadigital.org/revistacienciadigital2/index.php/CienciaDigital/article/view/250/553

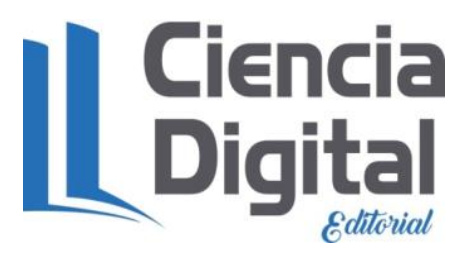

El artículo que se publica es de exclusiva responsabilidad de los autores y no necesariamente reflejan el pensamiento de la Revista Ciencia Digital.

El articulo queda en propiedad de la revista y, por tanto, su publicación parcial y/o total en otro medio tiene que ser autorizado por el director de la Revista Ciencia Digital.
